Protocols

\title{
Design and validation of an expeditious analytical method to quantify the emulsifying activity during biosurfactants/bioemulsifiers production
}

\author{
João Tavares ${ }^{1}$, Luís Alves *,1,2, Tiago P. Silva, Susana M. Paixão *,3 \\ LNEG - Laboratório Nacional de Energia e Geologia, IP, Unidade de Bioenergia e Biorrefinarias, Estrada do Paço do Lumiar, 22, 1649-038, Lisboa, Portugal
}

\section{A R T I C L E I N F O}

\section{Keywords:}

Analytical method

Emulsifying activity

Emulsification unit

Screening

Biosurfactant/bioemulsifier

Gordonia alkanivorans strain $1 \mathrm{~B}$

\begin{abstract}
A B S T R A C T
Biosurfactants (BS) and bioemulsifiers (BE) are amphiphilic molecules that are produced by a wide range of microorganisms. Although the chemical composition of BS and BE is different, both BS/BE have recognized emulsifying properties, which are the focus of this study. Herein, a rapid and simple analytical method to quantify the emulsifying activity (EA) of a product produced by the actinomycete Gordonia alkanivorans strain $1 \mathrm{~B}$ (BS/BE), which exhibits emulsifying properties, was developed. The analytical approach was based on the ability of a BS/BE solution to form a stable emulsion when mixed with n-heptane. So, using $4 \mathrm{~mL}$ screw cap glass tubes $(10 \times 75 \mathrm{~mm}$, ND10 caps with PTFE septum), the EA was assessed by adding $1 \mathrm{~mL}$ of $\mathrm{n}$-heptane to $1 \mathrm{~mL}$ of an aqueous solution containing the test product, mix by vortexing at high speed $(2 \mathrm{~min})$ and place the tube in an upright stable position for $10 \mathrm{~min}$ before analyzing. A set of emulsification tests with increasing volumes of test product solutions was carried out until $100 \%$ emulsion was obtained in the organic phase. One emulsification unit was defined as the minimum volume of product ( $\mathrm{Vol}_{\min }$ of emulsifier/surfactant, up to $1 \mathrm{~mL}$ ) needed to form and maintain $100 \%$ emulsion in the organic phase. The corresponding emulsifying activity value is presented in $\mathrm{U} / \mathrm{mL}$, and it is calculated as: EA (product) $=1 \mathrm{U} / \mathrm{Vol}_{\min }(\mathrm{mL})$. Further validation by testing several synthetic surfactants and industrial/domestic dishwashing detergents, in parallel with the bacterial crude BS/BE, towards emulsifying activity determination $(\mathrm{U} / \mathrm{mL})$ was performed demonstrating the wide range of the method applicability. Moreover, the specific emulsifying activity for each product tested was estimated though correlation analysis (linear regression) between volumetric emulsifying activity $(\mathrm{U} / \mathrm{mL})$ and product concentration $(\mathrm{g} / \mathrm{L})$. Indeed, this new analytical approach to quantify the emulsifying activity is accurate and reproducible, and consequently it can be a promising tool to apply in screening/monitorization studies on BS/BE production enabling reliable comparisons.
\end{abstract}

\section{Introduction}

To better explore their habitats, many microorganisms synthesize a broad range of amphiphilic substances, known as biosurfactants (BS) or bioemulsifiers (BE). According to Willumsen and Karlson [1], BS/BE are both surface active biomolecules, but while the surfactants play the role of surface tension reduction, emulsifiers are involved in formation and stabilization of emulsions. However, some biomolecules possess both surfactant and emulsifying properties, which contributes to their unique features, including high biodegradability, low toxicity, effectiveness at extremes of temperature, $\mathrm{pH}$ and salinity, and special biological activities (e.g. antimicrobial, antiviral, anticancer, etc). These attributes make them an alternative to their chemical counterparts and allows them to have key roles in several fields [2-6]. However, the biotechnological production of $\mathrm{BS} / \mathrm{BE}$ by microorganisms remains a great challenge in terms of productivity, quantities, and costs. But, considering its potential benefits and vast array of applications, its production is expected to reach industrial levels as soon as the economic obstacles are overcome, such as by introducing low-cost, renewable raw materials [2,7-10].

There are many screening methods to characterize biosurfactants, however, they are mostly based on the properties of these compounds to

\footnotetext{
* Corresponding authors.

E-mail addresses: luis.alves@lneg.pt (L. Alves), susana.alves@lneg.pt (S.M. Paixão).

1 These authors share first authorship.

2 ORCID ID: 0000-0001-6245-775X

3 ORCID ID: 0000-0003-0955-4467
} 